\title{
Study of transcranial colour Doppler in the measurement of cerebral edema in birth asphyxia
}

\author{
Jain $\mathbf{H}^{1}$, Arya $\mathbf{S}^{2}$, Thakur $\mathbf{K}^{3}$, Joshi $\mathbf{S}^{4}$ \\ ${ }^{1}$ Dr Hemant Jain, Professor, ${ }^{2}$ Dr Sunil Arya, Assistant Professor , ${ }^{3}$ Dr Kuldeep Thakur, P.G Student, ${ }^{4}$ Dr Swati Joshi, \\ P.G Student. All affiliated with Department of Pediatrics, M.G.M Medical College, Indore, MP, India
}

Address for Correspondence: Dr Sunil Arya, email- drsunilarya22@gmail.com

\begin{abstract}
Background: Perinatal asphyxia is an insult to fetus or newborn due to lack of oxygen /perfusion to various organs. Perinatal asphyxia occurs in 1 to 3 per 1000 live births and is related to gestational age and birth weight. Worldwide 4-9 million suffer from birth asphyxia. It occur in $9 \%$ of infants of $<36 \mathrm{wks}$ of gestation and $0.5 \%$ in $>36$ wks. Hypoxic ischemic brain injury is the most important consequence of perinatal asphyxia. Aim of study: To detect the measurement of cerebral blood flow in full term infants with birth asphyxia, by use of transcranial colour Doppler to measure RI (Resistive Index). Setting: Department of Paediatrics at MY hospital in collaboration with department of radiodiagnosis over a period of $1 \mathrm{yr}$ from November 2013- October 14. Design: Observational study. Method: It included 40 consecutive cases of birth asphyxia who suffered from HIE. Measurement of cerebral blood flow velocity was done by the use of colour doppler ultrasound. Result: Resistive index values were lower in measured vessels in asphyxiated infant as compared by blood flow velocity, resistive index of ACA and MCA. It is observed in our study that maximum number of patients in asphyxiated group belongs to grade II HIE $87.5 \%$ (35), while in stage III there were 2.5\% (1) and in stage I only 4 (10\%) patients. Conclusion: Colour Doppler USG would be of practical importance in evaluating the cerebral blood flow velocity in neonate with HIE. A skillful detection of decrease in cerebral blood flow of neonate in postnatal first $12 \mathrm{hrs} \&$ treatment being based on such detection would contribute to a good prognosis.
\end{abstract}

Key words: Resistive index, Birth asphyxia, Hypoxic ischemic encephalopathy, Colour Doppler

\section{Introduction}

Perinatal asphyxia refers to a condition during the first and second stage of labour in which impaired gas exchange leads to fetal hypoxemia and hypercarbia which in turn can lead to cerebral edema and various circulatory disturbances [1]. Asphyxia can be determined on the basis of indirect clinical markers, depressed Apgar scores, cord blood acidosis or clinical signs in the neonate due to hypoxic ischemic encephalopathy. Asphyxia is one of the leading causes of perinatal death and a recognized cause of neuromotor disability later in life among survivors [2].

During the post asphyxial period a consistent observation has been a marked increase in cerebral blood flow which continues for several hours and may or may not decline towards baseline. This reperfusion phase is responsible for secondary brain injury.

Manuscript received:10 ${ }^{\text {th }}$ March 2016

Reviewed: $24^{\text {th }}$ March 2016

Author Corrected: $11^{\text {th }}$ April 2016

Accepted for Publication: $23^{\text {th }}$ April 2016
In infants with HIE, high cerebral blood flow measured at 12 and 24 hours is associated with a poor neurological outcome [3].

Doppler sonography is a non invasive method and can help in early assessment of neonatal cerebral hemodynamics and shows consistent changes in cerebral blood flow velocities in infants with intrapartum asphyxia.

\section{Methodology}

It included 40 consecutive cases of birth asphyxia that suffered from HIE. Measurement of cerebral blood flow velocity was done by the use of colour doppler ultrasound. Cranial Ultrasound was done in neonates with birth asphyxia in first 24 hours of life and repeat scan on $3^{\text {rd }}$ postnatal day. Cerebral blood flow velocity recordings were made by colour duplex Doppler scanner 3 to $5 \mathrm{MHz}$. Recordings of CBF velocities were 
made in the supine position 30-60 minutes after feeding. Observations were made when the infants was in the quiet state, eyes closed and with no gross body movements. Anterior cerebral artery was visualized in the sagittal plane through the anterior fontanel and the signals were recorded from the point midway between the inferior-most border of the corpus callosum and the vessel origin from the circle of Willis. Middle cerebral artery was visualized through the temporal bone in the region above the zygomatic arch in the fold of the temporal lobe from the straight mid-portion of the artery. Neonates with Hypoxic ischemic encephalopathy included in this study were classified according to Sarnat $n$ Sarnat classification [4].

Measurement of Peak systolic velocity, End diastolic velocity and resistive index from anterior and middle cerebral arteries were conducted. In the present study we are taking an RI above 0.55 as normal and an RI value less than 0.55 as abnormal [5].

\section{Exclusion Criteria}

- Those infant who underwent ventilation treatment due to any reason.

- Those who are receiving medication such as dopamine, adrenaline.

- Those with major congenital anomalies or shock.

- Premature infants $<36$ weeks

Our study analysed the relationship of cerebral blood circulation parameters (PSV, EDV \& RI) registered in the anterior \& middle cerebral arteries evaluated at 12$24 \mathrm{hr}$ of life and at $3^{\text {rd }}$ post natal day and its relation to long term neurological outcome.

\section{Results}

1. Maximum numbers of asphyxiated infants were in grade-2- 35 patients $(87.5 \%)$ and remaining in grade- 1 (10\%) 4 patient and in grade-3 $(2.5 \%)$ only 1 patient.

2. Maximum number of cases of HIE were in birth weight between $2.5-2.9 \mathrm{~kg} 23(57.5 \%)$.

Table 1: Distribution of patients

\begin{tabular}{|c|c|c|c|c|}
\hline Grade & Male & \% & Female & \% \\
\hline HIE -1 & 4 & $10 \%$ & 0 & 0 \\
\hline HIE-2 & 24 & $60 \%$ & 11 & 0 \\
\hline HIE-3 & 1 & $2.5 \%$ & 0 & $27.5 \%$ \\
\hline TOTAL & 29 & $72.5 \%$ & 11 & \\
\hline
\end{tabular}

Male neonates involved more commonly than females.

This table shows mean resistive index of anterior and middle cerebral artery on $1^{\text {st }}$ day was $0.68 \pm 0.021$ and $0.53 \pm 0.052$ respectively, and on day $3^{\text {rd }}$ it was $0.55 \pm 0.061$ and $0.52 \pm 0.08$ respectively. There was no difference in mean value resistive index on day 1 and day 3 .

Table 2: Mean RI value in Anterior Cerebral artery according to grading of birth asphyxia

\begin{tabular}{|c|c|c|c|}
\hline & Number of cases & $\mathbf{1}^{\text {st }}$ day & $\mathbf{3}^{\text {rd }}$ day \\
\hline HIE-1 & 4 & $0.68 \pm 0.021$ & $0.55 \pm 0.061$ \\
\hline HIE-2 & 35 & $0.53 \pm 0.052$ & $0.52 \pm 0.08$ \\
\hline HIE-3 & 1 & 0.22 & 0.28 \\
\hline
\end{tabular}

This table show that means RI value in Middle cerebral artery decrease with severity of birth asphyxia, it is lowest in HIE-3, and in grade -2 . 
Table 3: Correlation of resistive index with grading of birth asphyxia

\begin{tabular}{|c|c|c|c|c|c|c|c|}
\hline Grade & Total & \multicolumn{3}{|c|}{ Abnormal } & \multicolumn{3}{c|}{ Normal } \\
\hline & & No. & $1^{\text {st }}$ day & 3rd day & No. & $1^{\text {st day }}$ & 3rd day \\
\hline HIE-1 & 4 & 0 & 0 & 0 & 4 & 0.62 & 0.56 \\
\hline HIE-2 & 35 & 35 & 0.53 & 0.55 & 0 & 0 & 0 \\
\hline HIE-3 & 1 & 1 & 0.22 & 0.53 & 0 & 0 & 0 \\
\hline
\end{tabular}

Resistive index was low in grade II and grade III HIE on day 1 and day 3 and was normal in HIE grade I.

\section{Discussion}

When asphyxia is followed by hypoxic ischemic injury to brain, a syndrome has been described known as 'Hypoxic ischemic encephalopathy'. Early assessment of the degree of resulting hypoxic- ischaemic encephalopathy (HIE) can provide prognostic information for both clinical management and the potential use of cerebroprotective strategies. However, clinical assessment is often difficult because the neurological state of the infant may be altered by pharmacological interventions such as sedation, muscle relaxation, or anticonvulsant treatment. Moreover, clinical signs of HIE may not develop until at least 12 hours after birth.

The haemodynamics in term infants with acute encephalopathy are deranged during the first 24 hours after presumed perinatal asphyxia. These consist of an increase in cerebral blood flow velocity (CBV) and a significant reduction in cerebral blood flow velocity resistance (CBVR). CBVR tended to return to normal values after the first 24 hours of age. $\mathrm{CBV}$ and $\mathrm{CBF}$ were frequently increased in the first 24 hours after birth were associated with a greater severity of acute encephalopathic signs and adverse outcome [6].

Attempts have been made to diagnose ischemic encephalopathy by use of CT, MRI and ultrasonography. Various authors in different study have emphasized that Doppler sonography is a practical, non-invasive and accurate method of diagnosing a wide spectrum of intra-cranial conditions due to HIE in neonate. Sonography is now established as a primary method for recognizing neonatal cerebral ischemia.

Having found normal RI values with Doppler method, the clinician can confidently reassure parents that their baby has a little risk of death. Prognostication of long term outcomes is one of the main objectives in Doppler sonography of the brain of full term neonate who experienced perinatal asphyxia. In this study maximum number of cases occurred in male. Maximum number of patients were weighing between $2.5-2.9 \mathrm{~kg} 23$ (57.5\%) in HIE-2. Ellis et al [7] \& Badani et al 2000 [8] in their studies observed that out of 144 infant 93 infant of weight $>2.5 \mathrm{~kg}$ suffered from grade -2 and 51 of weight between $2-2.5 \mathrm{~kg}$ suffered from mild asphyxia.

In our study out of 40 asphyxiated infants $77.5 \%$ (31) patients were born by vaginal delivery, and $22.5 \%$ (5) by LSCS.

Our study analyzed the relationship of cerebral blood circulation parameters (PSV, EDV \& RI) registered in the anterior \& middle cerebral arteries evaluated at 12$24 \mathrm{hr}$ of life and its relation to long term neurological outcome.

Doppler studies done in intracranial vessels in asphyxiated infants found an increase in diastolic flow and lowering of the RI to be the usual initial response detectable in initial 4 days of birth asphyxia[8].

In our study maximum cases were of HIE grade 2 and an abnormal RI on 1st and 3rd day was observed in $87.5 \%$ (35) cases ( $0.53-0.55$ in both MCA and ACA). sHowever further studies are needed to determine whether the cerebral blood flow velocity measurement in the intracranial artery is an accurate tool for assessing the severity of neurologic insult after birth asphyxia.

\section{Conclusion}

In the final course of our study, we have come to the conclusion that color Doppler USG would be of practical importance in evaluating the cerebral blood flow velocity in neonate with HIE.

A skillful detection of decrease in cerebral blood flow of neonate in postnatal first 12 hrs\& medication \& 
treatment being based on such detection would contribute to a good prognosis.

Early recognition of the hypoxic ischemic injury is important in guiding the management during those critical first days.

\section{Limitation}

1. Cerebral blood flow velocity was measured in intracranial artery (ACA ,MCA ) on day-1 and a repeat after 3rd day. However no repeat measurement was done on follow up.

2. The number of patient in this series, specially severely asphyxiated infants (HIE-3) is small because grade 3 infants have respiratory distress and their conditions often deteriorate after 2 nd day.

\section{References}

1. Paola Morales, Diego Bustamante, Pablo EspinaMarchant et al.Pathophysiology of perinatal asphyxia: can we predict and improve individual outcomes. EPMA J. 2011 Jun; 2(2): 211-230. doi: $10.1007 / \mathrm{s} 13167-011-0100-3$

2. Vannucci RC Hypoxic ischemic encephalopathy American Journal of perinatology DOI: 10.1055/s2000-9293

3. Judith H Meek, Clare E Elwell, David C McCormick, A David Edwards, Janice P Townsend, Ann L Stewart, John S Way ADC. fetal and neonatal edition- abnormal cerebral hemodynamics in perinatally asphyxiated infants and their outcome. Arch Dis Child Fetal Neonatal Ed. 1999 Sep; 81(2): F110-F115.

4. van Handel M, Swaab H, de Vries LS, Jongmans MJ. Long-term cognitive and behavioral consequences of neonatal encephalopathy following perinatal asphyxia: a review. Eur J Pediatr. 2007 Jul;166(7):645-54. Epub 2007 Apr 11.

5. Elstad M, Whitelaw A, Thoresen M. Cerebral Resistance Index is less predictive in hypothermic encephalopathic newborns. Acta Paediatr. 2011 Oct;100(10):1344-9. doi: 10.1111/j.16512227.2011.02327.x. Epub 2011 May 18.

6. Judith H Meek, Clare E Elwell, David C McCormick, A David Edwards, Janice P Townsend, Ann L Stewart, John S Way; abnormal cerebral hemodynamics in perinatally asphyxiated infants and their outcome. Arch Dis Child Fetal Neonatal Ed. 1999 Sep; 81(2): F110F115.

7. Badawi N, Kurinczuk JJ, Keogh JM, Alessandri LM, O'Sullivan F, Burton PR, Pemberton PJ, Stanley FJ. Intrapartum risk factors for newborn encephalopathy: the Western Australian case-control study. BMJ. 1998 Dec 5;317(7172):1554-.

8. van Bel F, van de Bor M, Stijnen T, Baan J, Ruys JH. Cerebral blood flow velocity pattern in healthy and asphyxiated newborns: a controlled study. Eur J Pediatr. 1987 Sep;146(5):461-7.

\section{How to cite this article?}

Vanza B, Patel U, Kulkarni, Khare N'Bilateral TMJ ankylosis, anesthetic and surgical challenge- case report. Pediatr rev. Int J Pediatr Res 2016;3(4):277-280. doi:10.17511/ijpr.2016.i04.13 